\title{
A GRADIENT ESTIMATE AT THE BOUNDARY FOR SOLUTIONS OF QUASILINEAR ELLIPTIC EQUATIONS
}

\author{
BY RONALD GARIEPY AND WILLIAM P. ZIEMER ${ }^{1}$ \\ Communicated by Hans F. Weinberger, April 8, 1976
}

1. Introduction. The purpose of this note is to present a result concerning regularity at the boundary of bounded, weak solutions of equations of the form

$$
\operatorname{div} A\left(x, u, u_{x}\right)=B\left(x, u, u_{x}\right)
$$

where $A$ and $B$ are, respectively, vector and scalar valued Baire functions defined on $\Omega \times R^{1} \times R^{n}$ that satisfy

$$
\begin{aligned}
& |A(x, u, w)| \leqslant a_{0}|w|^{p-1}+a_{1}|u|^{p-1}+a_{2}, \\
& |B(x, u, w)| \leqslant b_{0}|w|^{p}+b_{1}|w|^{p-1}+b_{2}|u|^{p-1}+b_{3}, \\
& w \cdot A(x, u, w) \geqslant c_{0}|w|^{p}-c_{1}|u|^{p}-c_{2} .
\end{aligned}
$$

Here, $\Omega$ is an open subset of $R^{n}, 1<p<n, c_{0}>0, a_{0} \geqslant 0, b_{0} \geqslant 0$, and the remaining coefficients are nonnegative, measurable functions in the respective Lebesgue classes

$$
\begin{aligned}
& a_{1}, a_{2} \in L_{n / p-1}(\Omega), \quad b_{1} \in L_{n / 1-\delta}(\Omega), \\
& c_{1}, c_{2}, b_{2}, b_{3} \in L_{n / p-\delta}(\Omega) \quad \text { where } 0<\delta<1 .
\end{aligned}
$$

A weak solution of $(1)$ is a function $u$ in the Sobolev space $W_{p}^{1}(\Omega)$ that satisfies $\int_{\Omega} A \cdot \nabla \varphi+B \cdot \varphi=0$ whenever $\varphi$ is a smooth function with compact support in $\Omega$.

It has been shown in [LU], [S], and [T] that a weak solution of (1) is Hölder continuous on compact subsets of $\Omega$. In connection with boundary regularity, it was established in [LSW] that a point $x_{0} \in \partial \Omega$ is regular for solutions of linear, uniformly elliptic equations in divergence form with bounded, measurable coefficients if and only if $x_{0}$ is regular for Laplace's equation. Later, Stampacchia [ST] extended this result to a wider class of linear elliptic equations. For solutions of quasilinear equations of the form $\operatorname{div} A\left(x, u_{x}\right)=0$, but subject to conditions more restrictive than (2), Maz'ya [M] established regularity at $x_{0}$ $\in \partial \Omega$ provided the following condition is satisfied:

AMS (MOS) subject classifications (1970). Primary 35D10, 35 B45; Secondary 35J25, $35 \mathrm{~J} 60,35 \mathrm{~J} 67$.

1 Research supported in part by a grant from the National Science Foundation. 


$$
\int_{0}^{1}\left[\Gamma_{p}\left(B\left(x_{0}, r\right)-\Omega\right) r^{p-n}\right]^{1 /(p-1)} \frac{d r}{r}=\infty .
$$

Here $B\left(x_{0}, r\right)$ denotes the $n$-ball of radius $r$ centered at $x_{0}$ and $\Gamma_{p}$ is a capacity defined on all sets $E \subset R^{n}$ by

$$
\Gamma_{p}(E)=\inf \left\{\int|\nabla f|^{p}\right\}
$$

where the infimum is taken over all $f \in L_{n p /(n-p)} \cap W_{p}^{1}\left(R^{n}\right)$ for which $E \subset$ int $\{x: f(x) \geqslant 1\}$. In view of the fact that $\Gamma_{2}$ is Newtonian capacity, one observes that (3) is precisely the classical Wiener condition when $p=2$.

2. The main results. Given a continuous function $f$ on $\partial \Omega$ and $x_{0} \in \partial \Omega$, we will say $u\left(x_{0}\right) \leqslant f\left(x_{0}\right)$ weakly for functions $u \in W_{p}^{1}(\Omega)$ provided that whenever $\eta$ is a smooth function supported in $B\left(x_{0}, r\right)$ and $f<k$ in $B\left(x_{0}, r\right) \cap \partial \Omega$, then $(u-k)^{+} \eta \in W_{p, 0}^{1}(\Omega)$. A similar definition is given for $u\left(x_{0}\right) \geqslant f\left(x_{0}\right)$ weakly and, therefore, we can give meaning to $u\left(x_{0}\right)=f\left(x_{0}\right)$ weakly.

As a direct consequence of the gradient estimate below, (5), we obtain the following

THEOREM. Suppose $f$ is a continuous function on $\partial \Omega$ and let $u \in W_{p}^{1}(\Omega)$ be a bounded weak solution of (1) such that $u\left(x_{0}\right)=f\left(x_{0}\right)$ weakly. If (3) holds and

$$
\int_{0}^{1}\left\|a_{1}+a_{2}\right\|_{n /(p-1), B\left(x_{0}, r\right)}^{1 /(p-1)} \frac{d r}{r}<\infty
$$

then $\lim _{x \rightarrow x_{0} ; x \in \Omega} u(x)=f\left(x_{0}\right)$.

The notation in (4) indicates the norm of $a_{1}+a_{2}$ taken relative to the $n$ ball $B\left(x_{0}, r\right)$. Of course, if it is assumed that $a_{1}, a_{2} \in L_{q}$ where $q>n /(p-1)$, then clearly (4) is satisfied.

It is interesting to observe that the regularity results of [LSW], [ST] , and [M] are obtained by employing potential-theoretic techniques, whereas ours is based primarily on information obtained from the differential equation itself. Indeed, the following estimate is the vital component.

If $u \in W_{p}^{1}(\Omega)$ is a bounded, weak solution of (1), let $\mu(r)=\sup \{u(x): x \in$ $\left.B\left(x_{0}, r\right)\right\}$, where $x_{0} \in \partial \Omega$. Suppose $k>f\left(x_{0}\right)$ and let $u_{k}=(u-k)^{+}$.

THEOREM. There is a constant $C$ depending only on $n, p$, the bound for $u$, the coefficients in (2), and $\delta$ such that for all sufficiently small $r$,

$$
r^{p-n} \int_{B\left(x_{0}, r / 4\right)}\left|\nabla u_{k}\right|^{p} \leqslant C[\mu(2 r)-\mu(r)+a(r)]^{p-1}
$$

whenever $u\left(x_{0}\right) \leqslant f\left(x_{0}\right)$ weakly and where 


$$
\begin{aligned}
a(r)= & r+\left\|a_{1}+a_{2}\right\|_{n /(p-1), B\left(x_{0}, r\right)}^{1 /(p-1)} \\
& +\left\|c_{1}+c_{2}+b_{2}+b_{3}\right\|_{n /(p-\delta / 2), B\left(x_{0}, r\right)}^{1 /(p-\delta / 2)}
\end{aligned}
$$

Suppose $u$ is a solution of (1) such that $u\left(x_{0}\right)=f\left(x_{0}\right)$ weakly but that $\lim _{x \rightarrow x_{0} ; x \in \Omega} u(x) \neq f\left(x_{0}\right)$. If (4) holds the gradient estimate (5) is used to show that there is a set $E$ which is $\Gamma_{p}$-thin at $x_{0}$ (see [ME] for definition) such that $u(x)$ tends to a limit as $x \rightarrow x_{0}, x \notin E$. Thus, in terms analogous to the classical case, $u$ has a $\Gamma_{p}$-fine limit at $x_{0}$.

Proofs of these and other results will appear elsewhere.

\title{
BIBLIOGRAPHY
}

[LSW] W. Littman, G. Stampacchia and H. F. Weinberger, Regular points for elliptic equations with discontinuous coefficients, Ann. Scuola Norm. Sup. Pisa (3) 17 (1963), 4377. MR $28 \# 4228$.

[LU] O. A. Ladyženskaja and N. N. Ural'ceva, Linear and quasilinear elliptic equations, "Nauka", Moscow, 1964; English transl., Academic Press, New York, 1968. MR 35 \#1955; 39 \#5941.

[M] V. G. Maz'ja, On the continuity at a boundary point of the solutions of quasilinear elliptic equations, Vestnik Leningrad. Univ. 25 (1970), no, 13, 42-55. (Russian) MR 43 \#706. 166.

[ME] N. Meyers, Continuity property of potentials, Duke Math. J. 42 (1975), 157-

[ST] G. Stampacchia, Le problème de Dirichlet pour les équations elliptiques du second ordre à coefficients discontinus, Ann. Inst. Fourier (Grenoble) 15 (1965), fasc. 1, 189-258. MR 33 \#404.

[S] J. B. Serrin, Jr., Local behavior of solutions of quasi-linear equations, Acta Math. 111 (1964), 247-302. MR 30 \#337.

[T] N. S. Trudinger, On Harnack type inequalities and their application to quasilinea elliptic equations, Comm. Pure Appl. Math. 20 (1967), 721-747. MR 37 \#1788.

\author{
DEPARTMENT OF MATHEMATICS, UNIVERSITY OF KENTUCKY, LEXINGTON, \\ KENTUCKY 40506
}

DEPARTMENT OF MATHEMATICS, INDIANA UNIVERSITY, BLOOMINGTON, INDIANA 47401 